\title{
Herausforderungen und Handlungsempfehlungen betrieblicher Weiterbildungspraxis in Zeiten der Digitalisierung
}

\author{
Malte Teichmann • André Ullrich • Julian Wenz • Norbert Gronau
}

Eingegangen: 16. Dezember 2019 / Angenommen: 31. März 2020 / Online publiziert: 16. April 2020 (C) Der/die Autor(en) 2020

Zusammenfassung Die Digitalisierung von Produktionsprozessen schreitet mit einer hohen Intensität voran. Weiterbildung hat eine hohe Relevanz für betriebliche Transformationsprozesse. Die betriebliche Weiterbildungspraxis ist den aktuellen Herausforderungen der Digitalisierung jedoch nicht gewachsen. Herausforderungen sind Kompetenzlücken der Mitarbeiter, ungewisse Anforderungsprofile und Tätigkeitstypen, demographischer Wandel sowie veraltete didaktische Ansätze. Zudem wird bestehender inhaltlicher und pädagogischer Freiraum bei der Gestaltung von Weiterbildung oftmals nur unzureichend ausgenutzt. Die skizzierte Situation führt dazu, dass der Mehrwert gegenwärtiger Qualifizierungsangebote sowohl für Unternehmen als auch Beschäftigte nicht ausgeschöpft wird. Ausgehend von Veränderungen durch Digitalisierung in der Produktion und deren Auswirkungen auf die Kompetenzentwicklung diskutiert dieser Beitrag Herausforderungen gegenwärtiger betrieblicher Weiterbildung. Er leitet Handlungsempfehlungen ab, die mithilfe von Beispielen gewerkschaftlich unterstützter Weiterbildungspraxis illustriert werden. Im Ergebnis erhalten Interessierte einen Überblick über gegenwärtige Herausforderungen und Handlungsempfehlungen für die Gestaltung und Durchführung von Weiterbildung in Zeiten der Digitalisierung.

Schlüsselwörter Betriebliche Weiterbildungspraxis · Digitalisierung von Produktionsprozessen · Gewerkschaftlich unterstützte Weiterbildungspraxis · Kompetenzentwicklung

\footnotetext{
M. Teichmann $(\bowtie) \cdot$ A. Ullrich $\cdot$ N. Gronau Universität Potsdam, Potsdam, Deutschland E-Mail: malte.teichmann@wi.uni-potsdam.de

J. Wenz

IG Metall-Bildungszentrum, Berlin, Deutschland
} 


\title{
Challenges and Recommended Actions for In-Company Vocational Training in Times of Digitization
}

\begin{abstract}
The digital transformation of production processes is constantly progressing. The human workforce is a central success factor, but employees must be prepared for the requirements induced by change, using inter alia competence development. In reality, however, the content-related and pedagogical freedom to design vocational training is often inadequately addressed. Based on the changes trough digitization in production processes, the challenges of current continuing vocational training in enterprises are discussed. Recommendations for action are then derived and illustrated by examples. The recommendations for action can serve as a basis for the design and implementation of their further vocational training practice.
\end{abstract}

Keywords Vocational training - Digitization of production processes $\cdot$ Labour union education $\cdot$ Competence development

\section{Ausgangslage}

Durch die Digitalisierung von Produkten, Produktionsprozessen, Produktionssystemen und der Arbeitsorganisation steigen die Anforderungen an die Mitarbeiter (Hirsch-Kreinsen 2014) sowie an organisatorische und didaktische Aspekte betrieblicher Weiterbildung. Technische Entwicklungen können in Gefahren für bestimmte Mitarbeitergruppen münden. Andere Gruppen sehen sich dagegen durch die Anreicherung von Arbeitsumgebungen mit erweiterten Tätigkeitsspektren konfrontiert (Spath et al. 2013). Beispielhaft sind hier der Wegfall von Hilfstätigkeiten in der Logistik durch Automatisierung der Lagerwirtschaft bzw. die Erweiterung des Kompetenzbereiches von Maschinenführern durch Vernetzung von Maschinen zu nennen.

Technologie-induzierte Transformationsvorhaben zielen auf die Erhaltung der Wettbewerbsfähigkeit von Unternehmen und der damit verbundenen Beschäftigungssicherung der Mitarbeiter ab. Für die erfolgreiche Transformation muss auch die Entwicklung der Mitarbeiter in den Fokus der Betrachtung rücken. Denn mit den neuen technologischen Möglichkeiten verändern sich die Arbeitsprozesse und damit einhergehend die Aufgaben der Beschäftigten, aus denen neue Kompetenzanforderungen resultieren.

Die Veränderungen betreffen mittelbar aber nicht nur die Kompetenzen der Mitarbeiter. Weiterbildung als Instrument der Kompetenzentwicklung zielt darauf ab, „Beschäftigungsfähigkeit zu sichern, Handlungsräume für Innovationsfähigkeit, Verantwortung und Leistung zu schaffen und darüber hinaus horizontale und vertikale berufliche Entwicklungswege zu ermöglichen“ (Gillen und Lindenkamp 2007, S. 235). Auch Weiterbildung muss sich folglich an die zukünftigen Anforderungen anpassen, um ihrer Aufgabe gerecht werden zu können. Didaktische Lehr- und Lernsituationen werden jedoch oft unter dem Leitbild einer Kompetenzvermittlung und daraus resultierendem Frontalunterricht realisiert, dessen Wirksamkeit lerntheoretisch und empirisch stark umstritten ist (u. a. Arnold 2018; Meueler 2018). 
Anders als die Ausbildung ist die betriebliche Weiterbildung nicht durch staatliche Rahmenbedingungen und Inhaltsvorgaben geregelt. Dies spiegelt sich in ihrer inhaltlich heterogenen Ausgestaltung wider, die quantitativ und qualitativ teilweise sehr unterschiedlich ausgeprägt ist (Meueler 2018). Um bedarfsgerechte Angebote zu realisieren, müssen Weiterbildungsverantwortliche Kenntnisse über den digitalen Wandel von Produktionsprozessen bzw. daraus resultierende Auswirkungen auf menschliche Arbeit haben. Sowohl erstere als auch letztere sind jedoch nur selten in der Praxis vorhanden (u.a. Faulstich 2018; Spath et al. 2013). Letztlich scheint es, als dass die praktische Umsetzung von Weiterbildung der erkannten Relevanz (u.a. IG Metall 2019a, 2019b) sowohl bei Inhalten als auch didaktischer Ausgestaltung in vielen Unternehmen hinterherhinkt.

Der Beitrag zielt darauf ab, für die skizzierte problematische Ausgangslage zu sensibilisieren, indem die Herausforderungen gegenwärtiger betrieblicher Weiterbildungspraxis herausgearbeitet werden. Weiterhin werden darauf aufbauend Handlungsempfehlungen für den zukünftigen Umgang gegeben. Zu diesem Zweck werden Veränderungen der Arbeitsprozesse durch Digitalisierung (Abschn. 2) sowie deren Auswirkungen auf Weiterbildung als Instrument der Kompetenzentwicklung (Abschn. 3) charakterisiert. Weiterführend werden Herausforderungen unterschiedlicher Themenbereiche betrieblicher Weiterbildungspraxis aufgezeigt (Abschn. 4). Anschließend werden den Herausforderungen Handlungsempfehlungen zugeordnet und diese durch Beispiele verdeutlicht (Abschn. 5). Abschließend wird ein Fazit gezogen (Abschn. 6).

\section{Produktionsprozesse im digitalen Wandel}

Die Veränderungen von Produktionsprozessen durch die Digitalisierung sind anhand der Schlagworte Dematerialisierung, Delinearisierung, Destandardisierung, Dehierarchisierung, Delokalisierung und Despezialisierung charakterisierbar (Gronau und Ullrich 2019): Dematerialisierung beschreibt die Überführung von vorher physischen Produkten und Objekten in digitale Produkte und Objekte. Neu ist, dass bei der Produktion die physische Form des Produkts nicht mehr im Vorhinein festgelegt sein muss. Beispielsweise kann an einen Kunden entweder ein Ersatzteil oder auch nur die 3D-Druck-Spezifikation des Ersatzteils gesandt werden.

Unter Destandardisierung wird verstanden, dass zwei Objekte oder Tätigkeiten, die früher gleich waren, jetzt individuell sein können. Diese Individualisierung wird durch Software und eingebettete Systeme erreicht, die nutzer- bzw. kundenspezifisch unterschiedliche Eigenschaften des Produkts zur Verfügung stellen.

Eine Folge der Destandardisierung ist auch die Delinearisierung von Arbeitsabläufen. Während bisher unterschiedliche Aufgaben der gleichen Kategorie in gleicher Art und Weise bearbeitet wurden, kann es zukünftig dazu kommen, dass die Aufgabenbearbeitung in unterschiedlicher Weise erfolgt, obwohl es sich um gleiche oder ähnliche Arbeitsergebnisse handelt. Beispielsweise kommunizieren die Werkstücke bei kurzfristigen Veränderungen mit den Maschinen, um zu erfragen, wo gerade Bearbeitungskapazitäten frei sind und beeinflussen so den Fertigungsprozess. 
Schließlich wird die Dehierarchisierung dazu führen, dass die bisherige Entscheidungsinstanz Hierarchie, die aus Menschen oder Funktionen von Informationssystemen besteht, in sehr starkem Maße durch eine direkte Koordination zwischen beteiligten Mitarbeitern und Objekten (Betriebsmitteln etc.) abgelöst wird. Die bisherige Frozen-Zone, das ist bei Serienherstellern der Zeitraum, in dem (teilweise mehrere Wochen lang) keine Änderungen der Produktkonfiguration möglich sind, wird deutlich verkürzt werden. Dies wird durch eine sehr viel direktere Kommunikation, beispielsweise zwischen dem Ausstattungslager, dem Zulieferer und der Endmontage, möglich. Die Dehierarchisierung erhöht den flexiblen Umgang mit kurzfristigen Änderungen.

Eine weitere Ausprägung der Digitalisierung ist als Delokalisierung zu bezeichnen. Durch die Verbindung der digitalisierten Elemente, z. B. eines Produktionssystems, über das Internet kann eine wesentliche Veränderung der Liefer- und Leistungsbedingungen weit außerhalb des eigentlich betrachteten Systems ausgelöst werden.

Risiken und Potenziale insbesondere für die Mitarbeiter entstehen durch die Despezialisierung. Die Einführung digitaler Assistenzsysteme (z. B. Einsatz einer Maschine mit Selbstregulierungsfähigkeiten wie statistische Prozesskontrolle) kann z. B. bei bestimmten Tätigkeitsprofilen zu einer Komplexitätsreduktion führen. Auch angelernte Beschäftigte werden somit bestimmte Aufgaben wahrnehmen können. Ein Verlust von Arbeitsplätzen ist dann zu erwarten, wenn Maschinen jene Aufgaben übernehmen, die vorher von Menschen gelöst wurden. Aus der Despezialisierung resultieren auch Anlässe zur Weiterqualifizierung Beschäftigter (z. B. Meisterausbildung für ältere Mitarbeiter) und gestiegene Nachfragen nach neuen Spezialisten für Aufgaben, die nicht durch automatisierte oder computerisierte Systeme (z. B. Überwachungs- und Steuerungsfunktionen, Kreativarbeit, IT-Spezialisten) abgebildet werden können.

\section{Auswirkungen der Digitalisierung auf Weiterbildung als Instrument der Kompetenzentwicklung}

Die Digitalisierung hat weitreichende Auswirkungen auf zukünftige Tätigkeitstypen und Kompetenzfacetten der Mitarbeiter. Dies führt zu erweiterten Kompetenzanforderungen, die sich ebenfalls stark auf Weiterbildung als Instrument der Kompetenzentwicklung auswirken.

Beispielhaft erhöht sich die Fehleranfälligkeit eines technischen Systems mit seiner Komplexität. Schematische Lösungen können nicht mehr im Vorhinein erlernt werden, da mögliche Fehler aufgrund ihrer Anzahl nicht mehr vorhersehbar sind. Beschäftigte, die stattdessen auf Basis von vorhandenem und abrufbarem Wissen auf Fehler reflexiv reagieren können, sind in diesem Zusammenhang unabdingbar (Gronau et al. 2017). Weiterbildung muss folglich den Erhalt und Ausbau von Wissen sowie die Entwicklung dieses reflexiven Handelns unterstützen.

Ebenfalls lösen sich konventionelle Arbeitsstrukturen und -organisationen durch den Anstieg digitaler Kommunikationstechnologien stetig auf. Berufliche und private Zeit überschneiden sich vermehrt. Gleichzeitig steigt der Interaktivitätsgrad von 
Arbeit, der sich u.a. in der räumlichen Trennung von Arbeitsprozess und -ergebnis (z.B. Maschinensteuerung durch Apps) sowie zunehmend verteilter Arbeit (z. B. virtuelle Meetings über Landesgrenzen hinweg) äußert. Diese räumliche und zeitliche Entgrenzung als auch Dehierarchisierung, Delinealisierung und Destandardisierung führen neben neuen Kompetenzbedarfen auch zu einem wachsenden Anspruch an die Eigenverantwortung und Flexibilität (Spath et al. 2013) der Mitarbeiter. Diese müssen durch Weiterbildung entwickelt werden.

Eine bedarfs- und zeitgerechte Allokation eigener Fähigkeiten innerhalb der Arbeitsorganisation und Wertschöpfungsprozessen hängt maßgeblich von individuellem Wissen des Einzelnen ab. Dies betrifft sowohl Wissen über ebenfalls an der Wertschöpfung beteiligte Organisationsentitäten und Prozesse als auch über Möglichkeiten und Grenzen eigener Ressourcen und Kompetenzen (Harteis et al. 2019). Letztere werden damit zu einem Gut, mit dem Mitarbeiter zunehmend wie ein Unternehmer wirtschaften müssen. Das notwendige Erkennen eigener Qualifizierungsbedarfe bzw. die Wahrnehmung von Potenzialen zur Kompetenzentwicklung erfordert eine verstärkte Selbstkontrolle, erweiterte Selbst-Ökonomisierung und Selbst-Rationalisierung des Individuums (Pongratz und Voß 2003). Erweiterte Handlungs- und Aufgabenspektren, gestiegene Kommunikations- und Interaktionsmöglichkeiten in der Arbeit sowie die schnell an Relevanz gewinnende Rolle des Arbeitskraftunternehmers sind damit weitere Faktoren, die innerhalb von Weiterbildung in den Fokus rücken müssen.

Neben der Befähigung der Mitarbeiter - fähig für den Wandel - müssen diese ebenfalls offen für bevorstehende Transformation - bereit für den Wandel - sein. Dementsprechend ist gleichermaßen die Entwicklung zum mündigen und selbstreflektierten Individuum zu fokussieren (Ullrich et al. 2015). Im Ergebnis führt die Digitalisierung zu immensen Anforderungen an die Kompetenzentwicklung der Beschäftigten, die durch betriebliche Weiterbildung adressiert werden müssen.

\section{Herausforderungen betrieblicher Weiterbildung}

Betriebliche Weiterbildung ist ein zentraler Bestandteil von Unternehmen. Planung und Durchführung der Weiterbildung sind durch gegebene strategische und strukturelle unternehmerische Rahmenbedingungen beeinflusst. Die operative Umsetzung von Weiterbildung wird mithilfe didaktischer Ansätze realisiert, die durch bestimmte Zieldimensionen und pädagogisches Handeln in konkreten Lehr-Lernsituationen gekennzeichnet sind. Ein Blick in die betriebliche Praxis zeigt, dass Weiterbildung in den genannten Themenbereichen vor Herausforderungen steht. Diese werden im Folgenden näher beschrieben.

\subsection{Verankerung in der Unternehmensstrategie}

Obwohl die Digitalisierung von Produktions- und Betriebsmitteln, Produkten und Geschäftsmodellen schnell voranschreitet, hinken viele Unternehmen bei der strategieorientierten Kompetenzentwicklung und Qualifizierung hinterher: Eine nachhaltige Personalplanung auf Basis systematischer Qualifizierungsbedarfsermittlung 
erfolgt oftmals nicht. Durch fehlende Profile bzgl. vorhandener Mitarbeiterkompetenzen als auch der mittel- und langfristigen Entwicklung des Betriebs bleiben notwendige Qualifizierungsbedarfe unentdeckt (IG Metall 2019b). Dies ist insbesondere vor dem Hintergrund der sich immer schneller wandelnden Tätigkeitsprofile und Kompetenzfacetten kritisch zu betrachten.

Ohne Erhebung und Analyse vorhandener Kompetenzprofile sowie einer anschließenden Ableitung konkreter Bedarfe bleibt die wichtige Rolle von Weiterbildung im Unternehmen unklar. Weiterbildungsangebote werden in dieser Folge eher als Kostenverursacher denn als notwendiger Beitrag für zukünftigen Unternehmenserfolg klassifiziert. Insbesondere in KMU läuft der digitale Wandel zudem langsamer als in großen Unternehmen an (Hirsch-Kreinsen 2014). Damit einher geht häufig ein fehlendes Bewusstsein für die Relevanz von Weiterbildung, da bisher auftretende Qualifizierungsbedarfe (z.B. durch Anschaffung einer neuen Maschine) mittels Anpassungsqualifizierungen adressiert werden konnten. Durch die Tragweite der Digitalisierung wird ein derartiges Vorgehen jedoch zukünftig nicht mehr ausreichen. Eine fehlende Bedarfsermittlung kann sich ebenfalls negativ auf die Nutzung öffentlicher Fördertöpfe auswirken. Einerseits werden diese aus Unwissen über Bedarfe oft nicht akquiriert. Andererseits steigt die Gefahr, dass Gelder mangels strategischer Planung für nicht relevante Weiterbildung genutzt werden.

\subsection{Organisation der Weiterbildung}

Aufgrund inhaltlicher Gestaltungsspielräume von Weiterbildung hängt die Qualität des darin adressierten Wissens maßgeblich von betriebsinternen Weiterbildnern ab, die oftmals sowohl für die organisatorische Planung als auch pädagogische Durchführung verantwortlich sind. Ebenfalls existieren keine standardisierten Qualitätssicherungsverfahren, um die pädagogische Qualität innerhalb betrieblicher Angebote zu gewährleisten. Zusammen mit fehlenden systematisch erhobenen Qualifizierungsbedarfen wächst damit die Gefahr für die Mitarbeiter und das Unternehmen, inhaltlich redundante Weiterbildungen durchzuführen. Diese Problematik verschärfend, verfügen nur wenige Beschäftige im Weiterbildungssektor über einen dezidierten erwachsenenpädagogischen Hintergrund. Gleichzeitig liegt für den pädagogischen Schwerpunkt der Erwachsenenbildung keine ,allseits bekannte und bewährte Didaktik“ (Meueler 2018, S. 1388) vor, die interessierten Weiterbildnern als Orientierungspunkt für die eigene pädagogische Arbeit dienen könnte.

Während in größeren Unternehmen Weiterbildung zumeist in eigenen Fachabteilungen oder als Teil der Human-Ressources institutionalisiert ist, fehlen in KMU häufig die dafür notwendigen zeitlichen, personellen und monetären Ressourcen. Weiterbildung wird dementsprechend, wenn überhaupt, nebenbei durchgeführt oder an externe Anbieter ausgelagert. Letzteres wird im Regelfall durch freiberufliche Coaches, Trainer und Dozenten realisiert. Jene Berufsbezeichnungen sind jedoch nicht gesetzlich geschützt, wodurch die inhaltliche und pädagogische Qualität unterschiedlich ausfallen kann. Im Ergebnis ist die Qualität betrieblicher Weiterbildung stark von den Unternehmensstrukturen abhängig. 


\subsection{Vereinbarung von Weiterbildungszielen}

Durch technische Entwicklungen wandeln sich bestehende Berufsbilder, die ihrerseits neue fachliche und persönliche Kompetenzen erfordern. Das Berufswissen von Personen, die länger in der gleichen Tätigkeit beschäftigt sind, nimmt somit kontinuierlich ab. Ebenfalls kann die Entwertung von Berufswissen die Gefahr von Arbeitslosigkeit erhöhen. Durch die immense Geschwindigkeit des Wandels gilt dies nicht ausschließlich für Berufserfahrene. Auch Berufseinsteiger sehen sich mit einer schnellen Alterung von in der Ausbildung vermitteltem Wissen und erworbenen Kompetenzen konfrontiert. Ebenfalls stellt die Akzeptanz digitaler Technologien (u.a. AR-Brillen, Robotern, intelligente Maschinen etc.) einen wesentlichen Erfolgsfaktor für den Wandel dar (Gronau und Ullrich 2019). Die Schaffung von Akzeptanz wird damit zur betrieblichen Querschnittsaufgabe, die auch die Planung und Durchführung von Weiterbildung beeinflusst.

Daran anschließend führt der gestiegene Einsatz von mobilen Endgeräten zu neuen Arten der Kooperation und Kollaboration bzw. zur Verfügbarkeit von Informationen in Echtzeit für jeden Mitarbeiter. Die im beruflichen Kontext genutzten Kommunikationstechnologien (bspw. Smartphones und Tablets) überschneiden sich stark mit jenen aus dem Privatleben. Dadurch ist mitunter eine hohe Akzeptanz gegenüber diesen spezifischen Kommunikationstechnologien gegeben. Mitarbeiter müssen aber auch für einen verantwortungsvollen Umgang, z. B. hinsichtlich des Datenschutzes, sensibilisiert werden. Zusammengenommen erweitern sich die Zieldimensionen betrieblicher Weiterbildung immens. Konventionell organisierte und durchgeführte Weiterbildung zeichnet sich jedoch primär durch Anpassungs- und Erweiterungsangebote aus, die punktuell identifizierte ,ad hoc“ Qualifizierungsbedarfe fokussieren. Vor dem Hintergrund der fehlenden systematischen Qualifizierungsbedarfsermittlung besteht hier Nachholbedarf.

\subsection{Schaffung geeigneter Lehr-Lernsituationen}

Gegenwärtig dominiert ein Kompetenzverständnis in Lehr-Lernsituationen, nach dem Kompetenzen durch äußere Einflüsse im Sinne eines steuerbaren Input-Output Verhältnisses vermittelbar seien. Hier wird eine Kausalität zwischen äußeren (Lehr-)Reizen (z. B. Instruktion durch einen Lehrenden) und internen (Lern-)Reaktionen der Lernenden unterstellt, die oftmals in konventionellem Frontalunterricht resultiert (u.a. Arnold 2018). Dessen Wirkungsgrad ist aus Perspektive verschiedener lerntheoretischer Ansätze jedoch fraglich. Die subjektorientierte Lerntheorie nach Holzkamp (1995) bspw. definiert derartiges Lernen als defensives Anpassungslernen, dessen Ziel in der Wiedergabe von Wissensfragmenten für die Erfüllung externe Erwartungshaltungen (z. B. in Form standardisierter Leistungstests) liegt. Paradox ist, dass dadurch äußere Anforderungen adressierbar sind. Gleichzeitig gelingt die Durchdringung von Wissensinhalten und daraus resultierender Kompetenzerwerb jedoch nicht, da primär die Wiedergabe und nicht das Verständnis von Wissen fokussiert sind.

Für Frontalunterricht wurde ebenfalls empirisch nachgewiesen, dass darin „erworbenes“ Wissen aufgrund fehlender Wissensreflexion langfristig abnimmt (u.a. 
Them et al. 2003). Ebenfalls ist zu bezweifeln, ob eine einseitige Informationsvermittlung ohne Reflexionsgelegenheit genug Lernmöglichkeiten bieten kann, bspw. komplexe Prozesszusammenhänge zu verstehen und daraus Rückschlüsse für den eigenen Arbeitsprozess abzuleiten. Durch den Wegfall einfacher Tätigkeiten sowie den fortschreitenden Fachkräftemangel wird ebenfalls eine Ausrichtung konkreter Lehr- und Lernsituationen auf bisher vernachlässigte Mitarbeitergruppen notwendig. Dies gilt insbesondere für an- und ungelernte sowie ältere Mitarbeiter, die mitunter Zielgruppenspezifika wie Lernblockaden, Akzeptanzprobleme oder lang andauernde Lernabstinenzen aufweisen.

\section{Handlungsempfehlungen für die betriebliche Weiterbildungspraxis}

Aus den Herausforderungen und aufbauend auf den Erfahrungen gewerkschaftlich unterstützter Weiterbildungspraxis lassen sich wesentliche Handlungsempfehlungen für gelingende betriebliche Weiterbildung ableiten. Zur Illustration der Handlungsempfehlungen werden ebenfalls praxisnahe Beispiele aufgezeigt.

\subsection{Themenbereich Unternehmensstrategie}

Grundlegend ist die Einführung einer systematischen Qualifizierungsbedarfsermittlung als Basis für eine zukunftsorientierte Personalplanung und -entwicklung zu empfehlen. Praktisch ist dies durch den Einsatz von Kompetenzmatrizen und -landkarten für Berufe und Tätigkeiten, systematische Qualifizierungsgespräche sowie einer Orientierung an den Tarifverträgen Qualifizierung und Bildung zu realisieren. Kompetenzmatrizen können u. a. einen Überblick über Kompetenzträger, deren Schlüsselpositionen sowie den wahrscheinlichen Zeitpunkt ihres Ausscheidens aus dem Unternehmen geben. Entstehende Kompetenzbedarfe können somit schnell identifiziert und rechtzeitig mittels Qualifizierung adressiert werden. Folgend wird, angelehnt an die Lösung der IG Metall (2017), beispielhaft dargestellt, wie bestehende Kompetenzen in einem Betriebsrat mithilfe einer Kompetenzmatrix (Abb. 1) festgehalten werden können.

Wesentlich für die Einführung einer systematischen Qualifizierungsbedarfsermittlung ist, dass Weiterbildung als Voraussetzung für den Betriebserfolg in Kultur

\begin{tabular}{|c|c|c|c|c|c|c|c|c|c|c|c|c|c|c|c|c|c|}
\hline \multicolumn{18}{|c|}{ Kompetenz- und Nachfolgematrix für Betriebsratsmitglieder } \\
\hline \multirow{2}{*}{$\begin{array}{c}\text { BR- } \\
\text { Mitglied }\end{array}$} & \multirow[b]{2}{*}{ Alter } & \multicolumn{7}{|c|}{ Amts- und Erfahrungszeiten } & \multicolumn{7}{|c|}{ Erwarteter Renteneintritt } & \multirow{2}{*}{$\begin{array}{c}\text { Schlüssel- } \\
\text { kompetenzen }\end{array}$} & \multirow{2}{*}{$\begin{array}{l}\text { Position in } \\
\text { Gremium X }\end{array}$} \\
\hline & & よ & 윰 & ঠ્ণ & \&્స & $\frac{\text { ก }}{\mathrm{N}}$ & 응 & ণ্ণি & ণ্ণ & ন্ত & ป్ & ฮิ & ปั & ๙ิ & ֻั & & \\
\hline Person A & 50 & & & & $\mathrm{X}$ & & $\mathrm{X}$ & $\mathrm{X}$ & & & & & & & & Kompetenz A & Position X \\
\hline Person B & 63 & $\mathrm{X}$ & $\mathrm{X}$ & & & $\mathrm{X}$ & $\mathrm{X}$ & $\mathrm{X}$ & & & $\mathrm{X}$ & & & & & Kompetenz B & Position X \\
\hline Person $\mathrm{C}$ & 65 & & & & & $\mathrm{X}$ & $\mathrm{X}$ & $\mathrm{X}$ & $\mathrm{X}$ & & & & & & & Kompetenz C & Position Y \\
\hline Person D & 25 & & & & & & $\mathrm{X}$ & $\mathrm{X}$ & & & & & & & & Kompetenz B & Position Y \\
\hline Person E & 30 & & & & & & & $\mathrm{X}$ & & & & & & & & Kompetenz A & Position Y \\
\hline$\ldots$ & .. & & & & & & & & & & & & & & & $\ldots$ & $\ldots$ \\
\hline
\end{tabular}

Abb. 1 Beispielhafte Darstellung einer Kompetenzmatrix (in Anlehnung an IG Metall 2017) 
und Strategie des Unternehmens verankert wird. Ein Kulturwandel kann durch verschiedene Sensibilisierungsmaßnahmen angeregt werden. Denkbar sind etwa die Erstellung eines Leitbildes Weiterbildung sowie die Durchführung von Sensibilisierungsworkshops, in denen z.B. an- und ungelernten Mitarbeitern Möglichkeiten zu Weiterqualifizierung im Unternehmen aufgezeigt werden. Ebenfalls können Tandems zwischen ehemaligen Weiterbildungsteilnehmern und an Weiterbildung interessierten Personen eingerichtet werden. Erstere können dadurch letzteren mit Ihren Erfahrungen als Ansprechpartnern z. B. bei fachlichen oder organisatorischen Fragen zur Verfügung stehen. Strategisch ist Weiterbildung dagegen in der Aufbauorganisation zu organisieren und zu institutionalisieren. Dies gelingt einerseits durch die Bereitstellung von monetären und zeitlichen Ressourcen, die in einem zuständigen Fachbereich gebündelt werden. Andererseits müssen personelle Ressourcen in Form von Arbeitsgruppen oder Einzelpersonen bereitgestellt werden, deren Aufgabe primär in der Planung, Organisation und Durchführung von Weiterbildung besteht.

Im Ergebnis sind Unternehmen durch die vorgeschlagenen Maßnahmen in der Lage, eine genaue Übersicht über vorhandene Kompetenzen im Betrieb zu erhalten, ggf. auf Basis der vorliegenden Daten Weiterbildungs- und Qualifizierungsbedarfe $\mathrm{zu}$ identifizieren und diese professionell umzusetzen. Beispielhaft ist hier der Fall einer dreijährigen Meisterausbildung zu nennen, die etwa durch die absehbare Berentung eines Mitarbeiters notwendig wird. Mit Hilfe einer systematischen Bedarfsermittlung ist der anstehende Bedarf rechtzeitig erkennbar. Daran anschließende Maßnahmen können geordnet durch die Weiterbildungsverantwortlichen organisiert werden. Ebenfalls ist durch die Konkretisierung des Bedarfssituation eine zielgerichtete finanzielle Förderung (z.B. auf Basis des Qualifizierungschancengesetzes) möglich. Ein Soll-Ist Gefälle zwischen notwendigen und vorhandenen Kompetenzen wird dadurch verhindert.

\subsection{Themenbereich Weiterbildungsorganisation}

Eine Weiter- und Nachqualifizierung der für die Organisation und Durchführung von Weiterbildung verantwortlichen Personen in den Themenfeldern Digitalisierung und Pädagogik hilft dabei, gegebene inhaltliche Gestaltungsspielräume optimal auf die Bedürfnisse des Unternehmens und der Mitarbeiter auszurichten. Für die fachliche Qualifizierung bieten sich Kooperationen mit erfahrenen Unternehmen (z.B. Betriebsbesuche) sowie Universitäten (z. B. in Projekten oder Abschluss von Weiterbildungsvereinbarungen) an. Pädagogische Kenntnisse dagegen, können in spezialisierten Programmen (z.B. „Train-the-Trainer“) erworben werden. Die Qualität externer Weiterbildung kann durch die Zusammenarbeit mit etablierten Trägern (z. B. der IG Metall) und Forschungseinrichtungen sichergestellt werden.

Eine thematisch auf die Digitalisierung ausgerichtete, qualitative Weiterbildung kann etwa in Lernfabriken geschehen, wie am Beispiel gewerkschaftlich unterstützter Weiterbildung illustriert wird. Diese wird durch die IG Metall u. a. mit der arbeitspolitischen Lernfabrik der Ruhr-Universität Bochum sowie dem Forschungsund Anwendungszentrum Industrie 4.0 (FAZI4.0) (Gronau et al. 2017) realisiert. Im FAZI4.0 werden z. B. Tagesschulungen zum Thema „Digitalisierung der Pro- 
Tab. 1 Ablauf einer Tagesschulung. (Eigene Darstellung)

\begin{tabular}{ll}
\hline Tagesschulung - „Auswirkungen von Industrie 4.0 auf die Arbeitszusammenhänge“ \\
\hline 8:30 Uhr & Begrüßung \\
8:45 Uhr & Praktische Darstellung konventionelle Produktion \\
& Praxisbeispiel „Herstellung eines Kniegelenkes“ im FAZI4.0 \\
& Produktionsabläufe, ,Nicht-intelligentes“ Werkstück, Manuelles Konfigurieren von \\
& Maschinen, Konventionelles Beheben von Produktionsstörungen etc. \\
9:15 Uhr & Impuls-Vortrag: ,,Technische Grundlagen der Industrie 4.0“ \\
& Grundlagen und Begriffsverständnis des Internet of Things, Chancen und Herausfor- \\
& derungen, Umsetzungsstand des Internet of Things in Deutschland, zugrunde liegende \\
& Konzepte im Internet of Things (u. a. Cyber-Physische Systeme), Einsatzbeispiele \\
& im unternehmerischen Kontext, Auswirkungen von CPS auf Fertigungsprozesse, Big \\
& Data und Neue Formen der Prozesssteuerung
\end{tabular}

9:45 Uhr

Kaffeepause

10:00 Uhr Impuls-Vortrag: „Auswirkungen auf die Arbeit“

Wandel der Arbeit und Rahmenbedingungen, Neue Tätigkeitsanforderungen und Kompetenzen, Fremdsteuerung und Handlungsspielraum der Tätigkeitstypen, Mensch-Maschine-Interaktion und Rolle von Erfahrungswissen

10:45 Uhr Vorführung und praktische Anwendung „Industrie 4.0 - Technologien“

Praktische Vorführung: AR-Brille, Kontextsensitive Informationsbereitstellung, Assistenzsysteme

Dezentrale Produktionssteuerung: Funktion, Möglichkeiten und Grenzen, Vor- und Nachteile für MitarbeiterInnen

11:30 Uhr

Mittagspause

12:30 Uhr

Entdeckendes Lernen: „Programmierung eines Raspberry Pi““

Demonstration von betrieblichen Szenarien mittels IoT

Anwendungsbeispiele (z. B. Nutzbarmachung von Sensordaten) aus dem Fertigungskontext

13:15 Uhr Interaktives Lernszenario: „Industrie 4.0 - Technologien in der Produktion“ Prozess „Einschleusen eines Eilauftrages in der smarten Produktion“

14:00 Uhr Kaffeepause

14:15 Uhr World Café - Fragen der Mitbestimmung im I4.0 Kontext \#1

Veränderungen der Tätigkeitsanforderungen, Rolle von Erfahrungswissen von Mitarbeitern, Gestaltungsmöglichkeiten, Mitbestimmungsrechte, Betriebliche Aus- und Weiterbildung

15:15 Uhr World Café - Fragen der Mitbestimmung im I4.0 Kontext \#2

Möglichkeiten der Mitgestaltung nach BetrVG und Herausforderungen der Arbeitsgestaltung

16:15 Uhr Evaluation

Evaluation der Veranstaltung durch Fragebögen

16:30 Uhr Schluss

duktion“ durchgeführt. Tab. 1 stellt den beispielhaften Ablauf einer Tagesschulung dar.

In der ersten Tageshälfte wird zielgruppenspezifisch aufbereiteter Input durch Lernbegleiter des FAZI4.0 gegeben. Die Inhaltsauswahl erfolgt auf Basis vorhandener Lerninteressen und der jeweiligen Betriebssituation. Steht ein produzierendes Unternehmen beispielsweise am Anfang des digitalen Wandels, herrschen hinsicht- 
lich potenziell drohender Jobverluste oft Unsicherheiten bei den involvierten Betriebsräten vor. Demensprechend werden u. a. die Grundlagen der smarten Produktionssteuerung als auch deren Auswirkungen auf menschliche Arbeit erläutert. Die in der ersten Phase erworbenen Wissensinhalte können in einer zweiten Phase mithilfe der Lernfabrik in praxisnahen Lernszenarien realitätsnah erlebt (z. B. Einschleusen eines Eilauftrages in der smarten Produktion) werden. Dabei entstehende Eindrücke werden anschließend mithilfe von Lernbegleitern dialogorientiert reflektiert (z. B. im Rahmen eines World-Cafés). Im Ergebnis können die Teilnehmer fachliches Wissen über die digitale Transformation als auch Handlungsfähigkeit erlangen.

\subsection{Themenbereich Weiterbildungsziele}

Die Digitalisierung hat massive Auswirkungen auf die Halbwertzeit von Ausbildungs- und Berufswissen. Dementsprechend sind mündige Individuen gefragt, die auch abseits zeitlich und inhaltlich organisierter Angebote eigenständig Kompetenzbedarfe ermitteln und adressieren können. Gelingende Weiterbildungspraxis muss folglich Angebote bereitstellen, die sowohl die Akzeptanz für selbstgesteuerte Kompetenzentwicklung steigern als auch den Erwerb von Techniken des selbstgesteuerten Lernens (z.B. Selbstreflexion, Recherche- und Lernmethoden) fördern. Der Anspruch an lebenslanges Lernen ist durch Unternehmen zu unterstützen. Letzteres gelingt u.a. mit der Bereitstellung spezifischer Lernräume, die sukzessive bei der Entwicklung von Lerntechniken helfen. Wesentlich bei deren Gestaltung ist eine enge Orientierung an den Bedürfnissen der Zielgruppe.

Beispielhaft wurde unter Mitwirkung der IG-Metall bei einem großen Zulieferer der Luftfahrtindustrie eine arbeitsorientierte Lernwerkstatt eingerichtet. Den Beschäftigten aus der Fertigung sollten damit leicht zugängliche Selbstlernangebote zur interessengesteuerten Kompetenzentwicklung verfügbar gemacht werden. Dafür wurde die Ausstattung der vorhandenen Ausbildungswerkstatt durch verschiedene Industrie 4.0-Technologien (u. a. kollaborierende Leichtbauroboter, 3D-Drucker inkl. Programmieroberfläche) ergänzt. Für den Einsatz des „LeanLab4.0“ wurde ein teilnehmerorientiertes didaktisches Konzept für Aus- und Weiterbildungsformate spezifischer Personengruppen entwickelt, in dem auch Abläufe, technologische Anwendungen und theoriegeleitete Begleitschulungen definiert sind. Durch Zusammenspiel von Lernumgebung und didaktischem Konzept ist es den Teilnehmern möglich, Lerntechniken sowie domänenspezifisches Wissen über digitale Technologien selbstgesteuert zu erlangen. Die Freude am Ausprobieren neuer Technologien und damit verbundener Erfolgserlebnisse sowie das Erkennen der Nützlichkeit steigert ebenfalls die Akzeptanz gegenüber diesen Technologien im eigenen Arbeitsprozess.

\subsection{Themenbereich Lehr-Lernsituationen}

Auf Basis verschiedener lerntheoretischer Ansätze sind Kompetenzen nicht im Sinne eines Input-Outputs vermittelbar. Dementsprechend ist ein Paradigmenwechsel vorzunehmen, der individuelle Kompetenzentwicklung bei der Planung und Durchführung von Weiterbildung als Leitbild positioniert. Lernprozesse sind dabei als 


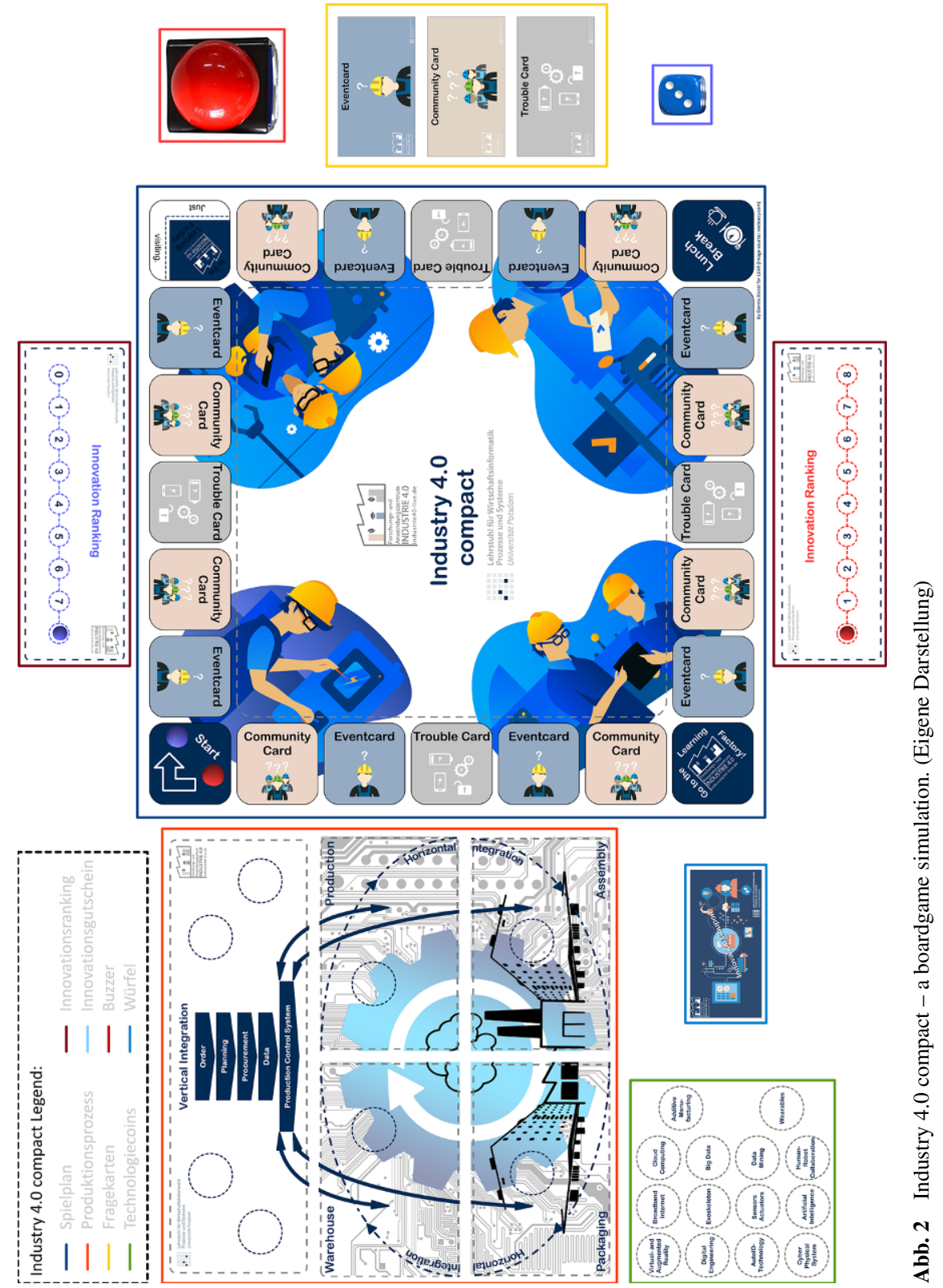


Tab. 2 Handlungsempfehlungen für gelingende betriebliche Weiterbildungspraxis

\begin{tabular}{|c|c|c|}
\hline $\begin{array}{l}\text { Themen- } \\
\text { bereich }\end{array}$ & Herausforderung & Handlungsempfehlungen \\
\hline \multirow[t]{4}{*}{$\begin{array}{l}\text { Unter- } \\
\text { nehmens- } \\
\text { strategie }\end{array}$} & $\begin{array}{l}\text { Zukünftige Qualifizierungsbedarfe sind } \\
\text { durch mangelhafte Personalplanung und } \\
\text {-entwicklung nicht antizipierbar }\end{array}$ & $\begin{array}{l}\text { Einführung einer systematischen Quali- } \\
\text { fizierungsbedarfsermittlung als Basis für } \\
\text { eine zukunftsorientierte Personalplanung } \\
\text { und -entwicklung }\end{array}$ \\
\hline & $\begin{array}{l}\text { Weiterbildung wird im Unternehmen als } \\
\text { vermeintlicher Kostenverursacher ver- } \\
\text { standen, da Qualifizierungsbedarfe nicht } \\
\text { erkannt werden }\end{array}$ & $\begin{array}{l}\text { Neben Einführung einer Qualifizierungs- } \\
\text { bedarfsermittlung ist Weiterbildung als } \\
\text { Voraussetzung für Unternehmenserfolg in } \\
\text { der Kultur und Strategie zu verankern }\end{array}$ \\
\hline & $\begin{array}{l}\text { In KMU fehlt oft das Bewusstsein für die } \\
\text { Relevanz von Weiterbildung, da die Trag- } \\
\text { weite des digitalen Wandels unterschätzt } \\
\text { wird }\end{array}$ & $\begin{array}{l}\text { Verankerung von Weiterbildung in der } \\
\text { Aufbauorganisation und Bereitstellung } \\
\text { monetärer, zeitlicher und personeller Res- } \\
\text { sourcen }\end{array}$ \\
\hline & $\begin{array}{l}\text { Fördertöpfe werden aufgrund verborgen } \\
\text { bleibender Qualifizierungsbedarfe nicht } \\
\text { genutzt }\end{array}$ & $\begin{array}{l}\text { Identifizierung möglicher Förderungs- } \\
\text { möglichkeiten auf Basis systematisch } \\
\text { erhobener Qualifizierungsbedarfe }\end{array}$ \\
\hline $\begin{array}{l}\text { Weiter- } \\
\text { bildungs- } \\
\text { organi- }\end{array}$ & $\begin{array}{l}\text { Betriebsinterne Weiterbildner haben nur } \\
\text { mangelhafte Kenntnisse über die Digitali- } \\
\text { sierung }\end{array}$ & $\begin{array}{l}\text { Weiter- und Nachqualifizierung im Bereich } \\
\text { der Digitalisierung durch externe Angebote } \\
\text { und Kooperationen }\end{array}$ \\
\hline
\end{tabular}

sation Eine fehlende Bedarfsermittlung führt zur Durchführung inhaltlich redundanter Weiterbildung

Die Weiterbildungsqualität externer Träger und Anbieter schwankt stark

Betriebsinternen Weiterbildnern fehlen pädagogische Kompetenzen

Ableitung von Qualifizierungsbedarfen aus der Unternehmensstrategie und Abstimmung der Inhalte

Zusammenarbeit mit etablierten Trägern und Anbietern

Weiter- und Nachqualifizierung im Bereich der Erwachsenenpädagogik durch interne oder externe Angebote

Fehlende monetäre, personelle und zeitliche Ressourcen in KMU verhindern betriebsinterne Weiterbildung

Weiter- Während Halbwertzeit von Berufswissen bildungs- sinkt, steigt die Gefahr diskontinuierlicher ziele Erwerbsbiografien durch Qualifikationsentwertung

Die Geschwindigkeit der Digitalisierung führt dazu, dass Ausbildungswissen und -kompetenzen schnell veralten

Die Akzeptanz des Wandels und dessen Technologien werden zur unternehmerischen Querschnittsaufgabe

Neue Arten des kooperativen und kollaborativen Arbeitens müssen durch WeiterbilKooperation mit externen Partnern (z. B. vergleichbaren Unternehmen, Gewerkschaften und Universitäten)

Bereitstellung von Angeboten und Ressourcen, die die Sensibilität der Mitarbeiter gegenüber selbstgesteuerten Kompetenzerwerb steigern und den Erwerb dafür notwendigen (Lern-)Techniken (Recherche, Lernmethoden etc.) ermöglichen

Zielorientierte inhaltliche Ausrichtung der Weiterbildung, um neues Wissen zu ergänzen und auszubauen

Akzeptanz als wesentliches Ziel positionieren und adäquate Angebote bereitstellen

Bereitstellung von Lehr- und Lernformaten, die ein konstruktives Arbeiten mit digitalen Medien fördern 
Tab. 2 (Fortsetzung)

\begin{tabular}{lll}
\hline $\begin{array}{l}\text { Themen- } \\
\text { bereich }\end{array}$ & Herausforderung & Handlungsempfehlungen \\
\hline $\begin{array}{l}\text { Lehr-Lern- } \\
\text { situa- } \\
\text { tionen }\end{array}$ & $\begin{array}{l}\text { Kompetenzvermittlung orientierter Frontal- } \\
\text { unterricht stellt das gegenwärtig dominie- } \\
\text { rende Format in betrieblichen Lehr-Lernsi- } \\
\text { tuationen dar }\end{array}$ & $\begin{array}{l}\text { Kompetenzentwicklung als Leitidee von } \\
\text { Lehr- und Lernsituationen verstehen und } \\
\text { darauf ausgerichtete mehrstufige Weiterbil- } \\
\text { dungsformate und -angebote schaffen }\end{array}$ \\
& $\begin{array}{l}\text { Zunehmend relevant werdende Zielgrup- } \\
\text { pen von Weiterbildung weisen bestimmte } \\
\text { Lernspezifika (z. B. Lernblockaden, Lern- } \\
\text { abstinenzen etc.) auf }\end{array}$ & $\begin{array}{l}\text { Bereitstellung niedrigschwelliger Angebote } \\
\text { zur sukzessiven Kompetenzanpassung und } \\
\text {-entwicklung, Entwicklung von Selbst- } \\
\text { wirksamkeit und Lernkompetenz (z. B. in }\end{array}$ \\
& $\begin{array}{l}\text { Lernfitnesstrainings) } \\
\text { Zielgruppenspezifische Akzeptanzproble- } \\
\text { me führen zu fehlender Weiterbildungsbe- } \\
\text { reitschaft }\end{array}$ & $\begin{array}{l}\text { Weiterbildung, die Widerstände als Aus- } \\
\text { gangspunkt für Akzeptanz- und Lernpro- } \\
\text { zesse aufgreift }\end{array}$ \\
& $\begin{array}{l}\text { Bestimmte Zielgruppen (z. B. An- und } \\
\text { ungelernte Mitarbeiter) nehmen bisher }\end{array}$ & $\begin{array}{l}\text { lung, die die berufliche und persönliche } \\
\text { bzw. überfachliche Entwicklung des Indivi- } \\
\text { selten an Weiterbildung teil }\end{array}$ \\
& &
\end{tabular}

subjektinterne Prozesse zu verstehen, die die selbstständige Wissensdurchdringung als Grundlage für den Kompetenzerwerb verstehen. Darauf aufbauend ist für die Planung ein bottom-up orientierter Ansatz zu empfehlen, der die Lernenden als einen zentralen Ausgangspunkt von Weiterbildung versteht. Dadurch können neben betrieblichen Qualifizierungsbedarfen auch Lerninteressen und Handlungsproblematiken der Teilnehmer von Anfang an in das Angebot integriert werden. Weiterbildung wird dadurch als mehrstufiger Prozess realisiert, der die persönliche Lernreise jedes einzelnen Mitarbeiters begleitet. Auch zielgruppenspezifische Besonderheiten (z. B. Lernblockaden oder Ängste) sind auf diese Weise adressierbar, indem bspw. Lernfitnesstrainings für ältere Mitarbeiter mit fachlicher Weiterbildung verbunden werden. Dieses Vorgehen schafft die Grundlage für die gleichmäßige Entwicklung fachlicher und persönlicher Kompetenzen (z. B. Selbstwirksamkeit und Lernkompetenz).

Darauf aufbauend sind Weiterbildungsformate zu kreieren, die die Reflexion und Anwendung von Wissen in neuen Problemkontexten ermöglichen. Aus didaktischer Perspektive bieten sich dafür u. a. problemorientierte oder Gamification-basierte Formate an, die Lernen durch Ausprobieren (z. B. in Lernfabriken) oder mittels Nutzung spielerischer Elemente (z. B. in Serious Games) ermöglichen.

Das Serious Game „Industry 4.0 compact - a boardgame simulation“ (Abb. 2) verdeutlicht, wie die Einbindung spielerischer Elemente in Lehr-Lernsituationen gelingen kann. Das Ziel des Spiels besteht darin, eine Produktion mittels technischer Innovationen zu digitalisieren. Dafür notwendige Innovationsgutscheine können durch lösen von Ereignis-, Community- oder Störungskarten erspielt werden. Auf den Karten gestellte Fragen und Aufgaben müssen auf bereits bekanntem Wissen oder neuen Lerninhalten (die z. B. während eines Lernszenarios in einer Lernfabrik erlernt wurden) aufbauen. Indem die Lernenden im Laufe des Spiels die Karten lösen, werden die in den Aufgaben adressierten Lerninhalte auf spielerische Art und Weise vertieft und gemeinsam reflektiert. Ebenfalls erfordert das kompetitive Spielziel eine enge Zusammenarbeit der unterschiedlichen Teams (z. B. bei der Lösung 
von Teamaufgaben), wodurch der dialogorientierte Wissensaustausch und -erwerb der Teammitglieder gefördert wird.

Ebenfalls möglich ist die Anreicherung des Arbeitsprozesses um Lernmöglichkeiten bzw. die Bereitstellung von am Arbeitsprozess orientierten Formaten. Beispielhaft ist ersteres durch Augmented-Reality gestützte Assistenzsysteme, letzteres durch den Besuch von Lernszenarien in Lernfabriken möglich. Mittels Assistenzsystem können etwa Lernaufgabe in die Arbeit implementiert oder dem Lernenden zusätzliche Wissensinhalte zur Verfügung gestellt werden.

Tab. 2 fasst die Herausforderungen und entsprechende Handlungsempfehlungen übersichtlich zusammen.

\section{Fazit}

Betriebliche Weiterbildungspraxis greift momentan oftmals zu kurz und steht vor strategischen und strukturellen Herausforderungen, die auf unsystematische Qualifizierung, technologie-induzierten Veränderungen der Arbeitswelt sowie den demografischen Wandel zurückzuführen sind. Gleichzeitig darf der pädagogische Anspruch an Weiterbildung nicht bei der fachlichen Ertüchtigung der Mitarbeiter enden, sondern muss auch auf die Entwicklung von Selbstständigkeit und Mündigkeit abzielen. Gelingende Weiterbildungspraxis hängt in dieser Folge stark von organisatorischen und didaktischen Aspekten ab, die positiv durch Unternehmen beeinflussbar sind. Wesentlich für den Erfolg ist das Erkennen von zukünftigen Qualifizierungsund Kompetenzbedarfen. Neben der praktischen Umsetzung in den Unternehmen ist gleichzeitig durch die Wissenschaft zu erforschen, warum die systematische Qualifizierungsbedarfsermittlung in der Praxis bisher nicht zum unternehmerischen Standard gehört. Ebenfalls sind, auch vor dem Hintergrund der steigenden Relevanz selbstgesteuerten Lernens, Mitarbeiter an Diskussionen über Veränderungen im Betrieb zu beteiligen. Indem individuelle Kompetenzentwicklung als Leitlinie von Weiterbildung positioniert wird, kann den Beschäftigten der dafür notwendige Mitgestaltungsspielraum bei ihrer eigenen qualifikatorischen Entwicklung ermöglicht werden.

Förderung Dieser Beitrag entstand im Rahmen der Arbeit der Forschungsgruppe 7 „Bildung und Weiterbildung in der digitalen Gesellschaft" am Deutschen Internet Institut (Kennzeichen 16DII116) sowie der Nachwuchsforschungsgruppe ProMUT „Nachhaltigkeitsmanagement 4.0 - Transformative Potentiale digital-vernetzter Produktion für Mensch, Umwelt und Technik“ (Kennzeichen 01UU1705B), die vom Bundesministerium für Bildung und Forschung gefördert werden.

Funding Open Access funding provided by Projekt DEAL.

Open Access Dieser Artikel wird unter der Creative Commons Namensnennung 4.0 International Lizenz veröffentlicht, welche die Nutzung, Vervielfältigung, Bearbeitung, Verbreitung und Wiedergabe in jeglichem Medium und Format erlaubt, sofern Sie den/die ursprünglichen Autor(en) und die Quelle ordnungsgemäß nennen, einen Link zur Creative Commons Lizenz beifügen und angeben, ob Änderungen vorgenommen wurden.

Die in diesem Artikel enthaltenen Bilder und sonstiges Drittmaterial unterliegen ebenfalls der genannten Creative Commons Lizenz, sofern sich aus der Abbildungslegende nichts anderes ergibt. Sofern das betreffende Material nicht unter der genannten Creative Commons Lizenz steht und die betreffende Handlung 
nicht nach gesetzlichen Vorschriften erlaubt ist, ist für die oben aufgeführten Weiterverwendungen des Materials die Einwilligung des jeweiligen Rechteinhabers einzuholen.

Weitere Details zur Lizenz entnehmen Sie bitte der Lizenzinformation auf http://creativecommons.org/ licenses/by/4.0/deed.de.

\section{Literatur}

Arnold R (2018) Das kompetente Unternehmen. Pädagogische Professionalisierung als Unternehmensstrategie. VS - Verlag für Sozialwissenschaften, Wiesbaden

Faulstich P (2018) Weiterbildung und Technik. In: Tippelt R, von Hippel A (Hrsg) Handbuch Erwachsenenbildung/Weiterbildung, 6. Aufl. VS - Verlag für Sozialwissenschaften, Wiesbaden, S 947-971

Gillen J, Linderkamp R (2007) Arbeitsnehmerorientiertes Coaching ein Ansatz zur Begleitung und Beratung beruflicher Entwicklungen im Betrieb. In: Dehnbostel P (Hrsg) Lernen im Prozess der Arbeit in Schule und Betrieb. Waxmann, Münster, S 233-246

Gronau N, Ullrich A (2019) Auswirkungen der Digitalisierung - Implikationen und Handlungsempfehlungen für Transformation und betriebliche Weiterbildung. In: Schröder M, Wegner K (Hrsg) Logistik im Wandel der Zeit - Von der Produktionssteuerung zu vernetzten Supply Chains - Festschrift für Wolfgang Kersten zum 60. Geburtstag. Springer, Berlin Heidelberg, S 471-494 https://doi.org/10. 1007/978-3-658-25412-4_22

Gronau N, Ullrich A, Teichmann M (2017) Development of the industrial IoT competences in the areas of organization, process, and interaction based on the learning factory concept. Proc Manuf 9:294-301. https://doi.org/10.1016/j.promfg.2017.04.029

Harteis C, Goller M, Fischer C (2019) Die Auswirkungen der Digitalisierung auf die Bedeutung beruflicher Qualifikation aus betrieblicher Sicht. In: Seifried J et al (Hrsg) Beruf, Beruflichkeit, Employability. wbv, Bielefeld, S 239-253

Hirsch-Kreinsen H (2014) Wandel von Produktionsarbeit - ,Industrie 4.0“. WSI-Mitteilungen 6: 421-429. https://doi.org/10.5771/0342-300X-2014-6-421. Zugegriffen: 7. April 2020

Holzkamp K (1995) Lernen: subjektwissenschaftliche Grundlegung. Campus, Frankfurt/Main und New York

IG Metall (2017) Praxistipps für den Generationswechsel. Nachfolgemanagement und Personalentwicklung Betriebsrat. Neues Druckhaus Dresden, Hamburg

IG Metall (2019a) Arbeit und Innovation. https://www.igmetall.de/arbeit-und-innovation. Zugegriffen: 5. März 2020

IG Metall (2019b) Transformationsatlas. Wesentliche Ergebnisse. Pressekonferenz der IG Metall. https://www.igmetall.de/download/20190605_20190605_Transformationsatlas_Pressekonferenz_ f2c85bcec886a59301dbebab85f136f36061cced.pdf. Zugegriffen: 5. März 2020

Meueler E (2018) Didaktik der Erwachsenenbildung/Weiterbildung als offene Projekt. In: Tippelt R, von Hippel A (Hrsg) Handbuch Erwachsenenbildung/Weiterbildung, 6. Aufl. VS, Wiesbaden, S $1385-1401$

Pongratz HJ, Voß GG (2003) From employee to 'entreployee'. Towards a 'self-entrepreneurial' work force? Concepts and Transformation. Concepts and Transformation 8(3):239-254. https://doi.org/ 10.1075/cat.8.3.04pon

Spath D, Ganschar O, Gerlach S, Hämmerle M, Krause T, Schlund S (2013) Produktionsarbeit der Zukunft - Industrie 4.0. Fraunhofer Verlag, Stuttgart

Them C, Schulca E, Ronerb A, Behrens J (2003) Comparison of frontal teaching versus problem-oriented learning at the school of healthcare and nursing: nursing neurological patients. Med Inf 71:117-124. https://doi.org/10.1016/S1386-5056(03)00096-0

Ullrich A, Vladova G, Thim C, Gronau N (2015) Akzeptanz und Wandlungsfähigkeit im Zeichen der Industrie 4.0. Prax Wirtschaftsinform 52(5):769-789. https://doi.org/10.1365/s40702-015-0167-8 